\title{
Sensibiliser les forestiers au paysage : ébranler les certitudes, construire ensemble de nouveaux savoirs
}

Philippe Deuffic

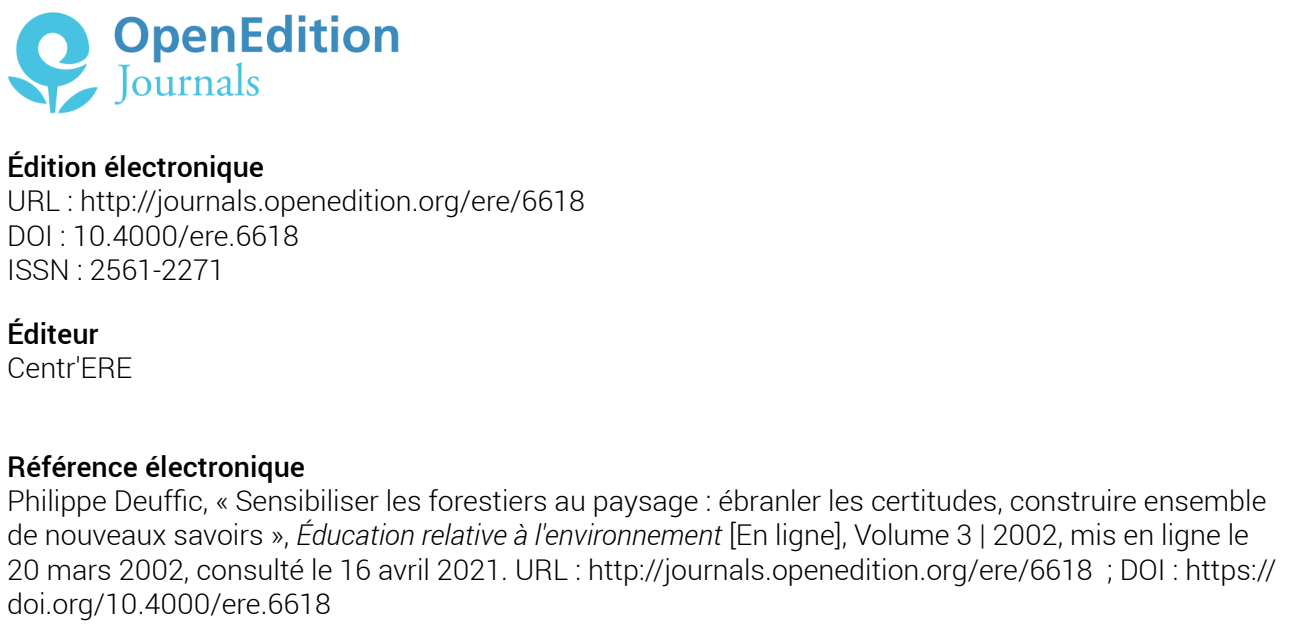

Ce document a été généré automatiquement le 16 avril 2021 


\title{
Sensibiliser les forestiers au paysage : ébranler les certitudes, construire ensemble de nouveaux savoirs
}

\author{
Philippe Deuffic
}

Depuis une dizaine d'années, les enjeux paysagers liés à la forêt prennent une place grandissante en France. D'une part, la forêt participe à la transformation des paysages ruraux, par expansion des boisements au sein des espaces agricoles. D'autre part, les regards que la société pose sur les forêts évoluent vite et sont parfois contradictoires : selon un sondage réalisé en novembre 2000 (SOFRES, 2001), $56 \%$ des Français préfèreraient une forêt entretenue et légèrement aménagée, $21 \%$ une forêt aménagée et comportant des espaces de loisirs, $20 \%$ une forêt sans aménagement et sans intervention humaine. Certains groupes sociaux dénoncent aussi, au nom du paysage, les essences très productives (résineux, peupliers), le mitage et la fermeture des espaces agricoles. Confrontés quotidiennement à ces questions, les organismes forestiers sont demandeurs de méthode permettant d'intégrer le paysage dans la gestion forestière. Nous verrons donc comment la Direction de l'Espace Rural et de la Forêt (DERF) du Ministère de l'Agriculture fait en sorte que ses agents intègrent cette problématique émergente dans leur pratique professionnelle. Cette action a démarré en 1994. Elle a été confiée au Cemagref, institut public de recherche en ingénierie de l'agriculture et de l'environnement, qui est engagé dans ces processus de réflexion sur les relations forêtspaysages depuis les années 1970. Cet article abordera successivement la nature de la commande passée par la DERF, ses objectifs et les différentes étapes du programme de sensibilisation conçu à cette occasion et les difficultés de partenariat lié au thème abordé et au public en formation. La spécificité d'un type de public - les agents forestiers - sera plus particulièrement abordée. 


\section{Le paysage, un enjeu croissant pour deux ministères}

2 À la fin des années 1980, le Ministère de l'Agriculture met en place des actions de sensibilisation au paysage à la demande des services forestiers en charge de la forêt privée. Mais la simple transposition des dispositifs techniques et paysagers développés pour la forêt publique dans les années 1970 s'avère inopérante en forêt privée. Non seulement les services forestiers alertent périodiquement la DERF de la persistance de problèmes paysagers, mais ils souhaitent aussi un positionnement plus clair du Ministère sur ces questions de paysage. Faute de véritable politique paysagère forestière (Luginbühl, 1993), mais consciente de la nécessité de prendre en compte cette problématique, la DERF lance un programme d'action à partir de 1994. Elle demande alors au Cemagref d'analyser pourquoi les réponses apportées jusqu'à présent en forêt privée fonctionnent peu et de cerner les questions et les attentes des agents forestiers notamment en matière de formation continue.

\section{Un état des lieux nécessaire}

L'étude menée auprès de ces services (Deuffic, 1996) montre :

- Une multiplicité et une dispersion des acteurs institutionnels intéressés par les questions de forêt et de paysage : onze ministères peuvent revendiquer d'avoir quelque chose à dire en matière de forêt ${ }^{1}$ et cinq ou six en matière de paysage. Pour autant, seuls deux ministères sont intéressés par les problèmes conjugués de la forêt et du paysage: le Ministère de l'Agriculture traditionnellement en charge des forêts et le Ministère de l'Environnement en charge des paysages. Chacun revendique sa prééminence sur le sujet, mais ni l'un ni l'autre n'ont de structure chargée simultanément des deux questions.

- Des difficultés de la part des agents à saisir les attentes des usagers : Les agents forestiers perdent progressivement le monopole de la compétence technique et administrative sur la forêt (Barthod, 1995). Celles-ci sont désormais investies par des groupes d'acteurs diversifiés et pour la plupart non forestiers : écologues, associations de protection de la nature et des paysages, associations d'usagers de la forêt, etc. Tous développent des relations sensibles à l'espace forestier dont les techniques d'analyses paysagères développées dans les années 1970 rendent compte imparfaitement. De fait, les agents forestiers disent éprouver des difficultés à dialoguer avec toujours plus de partenaires, à saisir des demandes qu'ils jugent floues et à intégrer la dimension sociale de la notion de paysage. Désappointés, certains d'entre eux n'abordent plus ces questions de paysage que sous l'angle de la technique sylvicole et les cantonnent aux seuls espaces boisés ; d'autres développent une vision picturale et pittoresque du paysage qu'ils assimilent trop hâtivement à une demande sociale (Freytet et Rumelhart, 2001).

- Des contenus de formation à adapter et à enrichir : Les personnels forestiers les plus âgés soulignent que leurs formations initiales ne comportaient pas ou peu de modules sur le thème du paysage. Quant aux formations continues, elles répondent partiellement à leurs attentes. Leurs contenus sont très axés soit sur les techniques d'aménagements sylvicoles et paysagers, soit sur la dimension esthétique et sensible propre à un professionnel (en l'occurrence le paysagiste formateur). Les relations sensibles et les enjeux des groupes sociaux concernés localement sont plus rarement abordés.

- Une incompréhension mutuelle entre forestiers et professionnels du paysage : les agents forestiers trouvent peu de professionnels du paysage capables de comprendre leurs 
problèmes de sylviculture. Les paysagistes professionnels et les chargés de mission " paysage » des services déconcentrés du Ministère de l'Environnement sont souvent peu formés, voire sensibilisés à la sylviculture et à son langage technique très spécifique. Pour autant, certains prennent quand même position sur la forêt et la sylviculture sans toujours bien maîtriser les processus sylvicoles en jeu (Freytet et Rumelhart, 2001). De fait, à force d'incompréhension et d'absence de langage commun, ces deux catégories de professionnels de la forêt et du paysage travaillent souvent chacune de leur côté.

\section{La mise en place d'une formation commune, une invitation au partenariat}

4 À l'issue de cette étude, deux axes d'interventions majeurs sont retenus :

- La DERF demande au Cemagref de mettre en place un cycle de sensibilisation au paysage à l'attention de ses agents. À l'issue de ces formations, la DERF souhaite qu'un groupe d'échange d'information autour du thème "Forêts-Paysages » se constitue sur la base des agents sensibilisés.

- La DERF propose à la Direction de la Nature et des Paysages (DNP), qui dépend du Ministère de l'Environnement, d'ouvrir les formations à ses personnels des services déconcentrés. L'idée est de faire se rencontrer les agents des deux ministères afin qu'ils échangent leurs points de vue sou-vent opposés sur cette thématique "Forêts-Paysages ». Le temps de la formation est alors conçu comme un moment privilégié pour débattre, en dehors des enjeux institutionnels quotidiens, d'un thème pour lequel la construction et la valorisation réciproque des savoirs paraît a priori envisageable.

\section{La problématique d'une formation au paysage}

5 Si l'état des lieux mené en 1995 témoigne de l'intérêt de sensibiliser les agents forestiers à la problématique "Forêts-Paysages ", l'objet même à traiter - le paysage et le public à former - des professionnels - compliquent l'élaboration du programme pédagogique.

\section{Spécificité du contenu pédagogique La diversité des définitions}

6 La difficulté avec la notion de paysage - mais aussi son intérêt - réside dans le fait qu'il s'agit d'une notion polysémique. Pour le géographe, A. Berque (1995), il y a dans notre langue deux acceptions très différentes du terme paysage « dans la première, celle du paysage grandeur nature, il s'agit de la réalité au premier degré, telle qu'elle apparaît directement à nos sens, mais dans la seconde acception, le paysage est une image, une représentation des choses en leur absence ". Les définitions du paysage sont donc multiples et se partagent selon quatre grandes familles d'approches conceptuelles: le paysage comme espace naturel ou espace anthropisé et le paysage comme image résultant d'une activité perceptive individuelle ou comme construction mentale socialement cadrée (Ragouet, 2000). De fait, les travaux sur le paysage empruntent des méthodes à la géographie physique, l'écologie, mais aussi à la géographie sociale, à la sociologie, l'anthropologie. Ce foisonnement des approches rend difficile la mise en place d'un programme de sensibilisation. Il est aussi à l'origine des incompréhensions des personnels en butte à des problèmes d'ordre paysager. 


\section{Une notion nécessairement subjective}

7 Une question fréquemment posée par les agents touche à la subjectivité et à la dimension de «représentation» de la notion de paysage. Pour Berque (1995), cette ambiguïté du terme paysage « vient de ce qu'elle concerne directement notre rapport à la réalité. Elle nous enseigne que ce rapport lui-même est ambivalent. Ce que nous voyons de l'environnement réel, un paysage, ne peut en effet pas être complètement dissocié de la manière dont nous nous le représentons ». Or, la tentation est grande pour les personnels de terrain de se réfugier dans des démarches d'analyses paysagères dites objectives et de réfuter toute subjectivité pourtant inhérente à la notion de paysage. Leur souci est d'aboutir à une analyse paysagère incontestable qui permette d'agir rapidement et qui soit traduisible en termes techniques. La plupart d'entre eux n'intègrent donc pas - ou peu - la dimension sociale des questions de paysage.

Or, selon Berque (1995) :

le paysage n'est pas un objet. Pour le comprendre, il ne suffit pas de savoir comment s'agencent morphologiquement les constituants de l'environnement ni comment fonctionne la physiologie de la perception (...), il faut connaître aussi les déterminations culturelles, sociales et historiques de la perception - autrement dit, ce qui construit la subjectivité humaine.

La notion de paysage déborde ainsi largement l'espace forestier et sa dimension technique. Elle s'étend à l'ensemble des composantes paysagères de l'espace rural. Prendre en compte le paysage signifie qu'il faut à la fois comprendre les pratiques et les modes d'appropriation de l'espace des propriétaires forestiers privés et des autres usagers, d'intégrer leurs représentations paysagères et les liens sensibles qu'ils tissent avec l'espace, de négocier entre des enjeux contradictoires, etc. Or les sciences humaines offrent des méthodes et des outils permettant de saisir cette subjectivité via l'étude des représentations sociales du paysage (Luginbühl, 1991) et les approches ethnologiques (Coll., 1995) ou sociologiques (Coll. 1991 ; Micoud, 1991).

\section{Spécificité du public en formation}

\section{Une sensibilisation moins technique, plus sociale}

10 Une autre spécificité de ce programme tient au fait que la formation s'adresse à un public adulte. À la différence des jeunes en formation initiale, le public adulte vient en formation avec une expérience professionnelle significative, des références construites dans un univers professionnel plus ou moins perméable et une personnalité sociale établie (Dubar, 2000). Dans le cas des forestiers, la formation initiale, l'expérience professionnelle et les formations continues se font dans des structures qui ont toutes une dimension "forestière » clairement affichée (lycées, organismes professionnels, services internes, centres de formation professionnelle, etc., " forestiers »). Il est alors plus difficile de modifier voire d'inverser des gestes techniques ou des idées établies de longue date et partagées par l'ensemble du corps professionnel. Un enjeu important de la formation consiste à infléchir voire modifier des savoirs et des pratiques professionnels sans les déstructurer ni les dévaloriser.

11 La réceptivité des agents à la diffusion d'un nouveau message peut aussi être affaiblie par le contenu de la formation qui désoriente le stagiaire. Vue la polysémie de la notion de paysage, celle-ci peut être traitée de manière très différente d'une structure de 
formation à une autre. Comment alors intégrer et valoriser ces expériences de terrain et aider le stagiaire à s'y retrouver entre différents contenus pédagogiques acquis lors de stages antérieurs? L'identification préalable des expériences et des formations suivies par l'agent est donc nécessaire. Elle permet de faire le point avec lui sur les interprétations complémentaires ou divergentes qui entourent des notions complexes comme celle de paysage.

Si le public professionnel vient en formation continue avec des questions, il aime aussi à repartir avec des réponses voire des certitudes et si possible des itinéraires techniques facilement applicables. Cela n'est pas toujours possible ni souhaitable. Sensibiliser à l'environnement comme au paysage, c'est introduire de la complexité dans la compréhension des phénomènes. Quels savoirs va-t-on alors proposer au stagiaire ? Est-il forcément utile qu'il résolve lui-même les problèmes dans toutes leurs dimensions?

Cela pose donc la question des objectifs assignés à la formation par l'employeur qui envoie son personnel en stage et les propres objectifs du stagiaire. Il convient que les deux parties se mettent d'accord sur la valorisation des nouvelles connaissances acquises et sur les moyens de les mettre en œuvre. S'agit-il pour le commanditaire de transformer son agent forestier en spécialiste du paysage? S'agit-il simplement de le sensibiliser au problème, de lui donner les moyens d'identifier les situations de conflit autour de la notion de paysage, de mobiliser les compétences nécessaires à l'extérieur du service, etc. ?

\section{La structure de la formation}

\section{Les objectifs du programme}

Le cycle de sensibilisation aux problèmes de paysages mis en place par le Cemagref à la demande de la DERF a pour objectifs de :

- Sensibiliser de manière approfondie à la question du paysage forestier des agents des services déconcentrés en charge de la forêt ou de l'environnement. L'idée essentielle est que des agents de ces services partagent des éléments de langage ou de «culture » communs. Pour les forestiers, partant d'une absence de "culture paysagère » initiale, il s'agit de leur donner une «culture de base» suffisante pour dialoguer avec des paysagistes professionnels ${ }^{2}$. Pour les agents des services déconcentrés du Ministère de l'Environnement, il s'agit d'appréhender le langage et les contraintes techniques propre à la sylviculture.

- Favoriser les échanges d'informations et d'expériences sur ce thème entre agents de différentes régions, entre agents intervenant dans des institutions différentes, et enfin entre chercheurs, formateurs et agents de terrain.

\section{Les formateurs et les moyens matériels}

Pour aborder cette problématique du paysage sous des angles différents, des professionnels de divers horizons interviennent: des paysagistes publics et libéraux, des responsables de services forestiers et de Parcs Naturels Régionaux, des juristes, des sociologues, des enseignants des écoles de paysage, des agents de développement chargés de la mise en place de Plans de Paysage, etc. L'animation et la coordination des intervenants sont assurées par le Cemagref. L'organisation matérielle se fait dans le 
cadre d'un Centre de Formation Professionnelle et de Promotion Agricole (CFPPA) sous tutelle du Ministère de l'Agriculture.

\section{Un programme de formation en trois étapes}

16 Cette formation de 80 heures est structurée en trois sessions de quatre jours étalées sur un an et situées dans trois contextes paysagers et forestiers différents. Le public concerné étant adulte, provenant de deux grands types d'horizons professionnels, l'un forestier, l'autre environnementaliste, et évoluant dans des zones géographiques différentes, le choix d'une pédagogie active a été opéré d'emblée par les coordinateurs de la formation. Plutôt que la seule transmission de savoir qui risque de décourager les stagiaires, la formation privilégie la participation des agents lors de travaux pratiques appelés sites-ateliers. L'objectif de ces sites-ateliers est de recréer des situations d'enjeux paysagers aussi proches que possible de celles rencontrées au cours de leur activité professionnelle et d'en discuter entre membres du groupe. Les choix pédagogiques qui sous-tendent la formation visent donc à reconnaître les savoirs acquis et les expériences de terrain, à laisser une large place à l'apprentissage à partir de cas concrets, à favoriser les discussions collectives en vue de résoudre le problème posé, l'ensemble étant articulé avec des apports théoriques plus formels.

\section{Session $1:$ Introduction à la notion de « paysage »}

17 L'étude préalable au programme de formation avait montré une grande hétérogénéité des niveaux de sensibilisation des agents à la question du paysage. La première session fait donc le point avec les stagiaires sur l'origine de la notion de paysage, ses différentes interprétations, son incontournable complexité et ses implications dans le domaine de l'aménagement de l'espace. Les formateurs et des paysagistes professionnels introduisent aussi le vocabulaire spécifique utilisé lors des analyses paysagères. Des méthodes de lecture du paysage par approche visuelle et sensible sont testées par les stagiaires sur le terrain lors de sites-ateliers. Celles-ci s'inspirent des méthodes de diagnostic des territoires mises en œuvre dans à l'occasion du Programme Européen de Formation Agri-environnement (ENPDD, 1995). Elles permettent aux stagiaires de confronter leur vision d'un paysage à celle des autres membres du groupe. Ces exercices leur permettent de mobiliser à la fois des approches dites "objectives » et d'autres "subjective ». La confrontation de ces deux approches, dès la première session, que beaucoup de stagiaires redoutent, permet de dédramatiser cet aspect de l'analyse paysagère.

\section{Session 2 : Paysage et aspects sociaux}

La deuxième session montre comment les perceptions et les représentations sociales des paysages de différentes catégories d'acteurs peuvent être prises en compte. L'accent est mis sur la diversité de ces représentations, des enjeux et des stratégies propres à chaque groupe d'acteurs. Cette session a pour objectif essentiel de montrer qu'il est possible d'intégrer des données dites "subjective " dans une démarche d'analyse paysagère. Elle présente quelques méthodes d'enquêtes issues des sciences humaines capables de prendre en compte ces données. À l'occasion du site-atelier, les stagiaires sont donc invités, en tant que conseillers forestiers, à établir un diagnostic 
sur un territoire où il existe une forte dynamique des paysages forestiers et agricoles. En fin d'exercice, ils proposent divers scénarios d'aménagements agricoles et forestiers sur une partie de la commune étudiée. À la différence de la première session, ils doivent aussi intégrer dans leur analyse la dimension sociale et culturelle que les différents groupes d'acteurs locaux affectent à ce paysage, à la forêt et prendre en compte leurs diverses revendications. Outre la diversité des représentations sociales du paysage, les agents touchent ici du doigt la diversité des représentations qui existe aussi à propos de la forêt.

\section{Session 3 : Les techniques paysagères}

19 La DERF ne souhaitait pas, à l'origine, que les personnes sensibilisées interviennent directement dans l'élaboration de solutions techniques. Néanmoins, la demande des agents sur ce point était forte. Une troisième session explore donc les techniques d'aménagements paysagers appliquées au milieu forestier (Breman, 1993 et 1997). Au cours de celle-ci, en principe, seules des questions techniques sur l'intégration paysagère des opérations forestières (coupe d'exploitation, éclaircie, création de piste forestière, etc.) sont abordées. En fin d'atelier, un projet sylvicole et paysager est présenté au propriétaire de la parcelle concernée.

\section{Premiers résultats, premiers enseignements}

20 Les sessions de formation « Forêts-Paysages ", fonctionnent depuis trois ans. Elles ont été suivies par 51 agents.

\section{Les points acquis}

21 S'il est un point qui conditionne particulièrement le succès de ces formations - certes encore à évaluer - c'est l'analyse préalable des besoins des stagiaires. Comme le constate Dubar (2000), trop souvent l'offre de formation préexiste à l'inscription des personnels qui doivent choisir leur stage à partir d'éléments vagues (un titre, une durée, des thèmes). Même lorsque le stage a été construit à partir d'une analyse des besoins, ce sont rarement les stagiaires qu'on a consultés. Leurs besoins ont été définis en dehors d'eux, par des décideurs qu'ils ne connaissent pas. Or, dans le cas précis de la formation proposée par la DERF, cette analyse des besoins s'est faite bien avant la mise en place des stages. Elle a été effectuée auprès soit de futurs stagiaires soit de leurs confrères évoluant dans les mêmes structures professionnelles et non définie seulement entre le commanditaire et le responsable de la formation.

Quant au programme pédagogique lui-même, ses avantages sont multiples. Premièrement, l'acquisition du langage spécifique aux études paysagères permet aux forestiers de mieux comprendre les paysagistes professionnels. Cette formation permet aussi aux agents de pouvoir apprécier la nature et la qualité des études paysagères réalisées en milieu rural et en forêt en particulier. La formation n'est donc pas conçue pour leur permettre de réaliser par eux-mêmes des études paysagères complexes. On rejoint là une conception de l'État qui a la charge d'orienter l'action publique, mais pas de se substituer aux bureaux d'études privées. 

inhérente au paysage et sa prise en compte comme critère normal d'analyse préalable aux actions sylvicoles. À titre d'exemple, bien que la troisième session soit centrée sur l'adaptation des techniques sylvicoles, les stagiaires se sont interrogés d'emblée sur les diverses réactions possibles des groupes sociaux concernés par l'opération sylvicole qu'ils menaient sur la commune. Cela témoigne d'un changement de posture professionnelle majeur: ils prennent conscience que malgré leur savoir en matière forestière ou environnementale, ils n'ont pas a priori plus de légitimité que d'autres groupes sociaux pour définir ce que doit être le paysage. salle apportent les éléments théoriques indispensables à la compréhension du sujet traité, les travaux pratiques présentent un aspect concret et démonstratif pour les stagiaires. Enfin, si l'objectif n'est pas que les agents manipulent par eux-mêmes les méthodes d'analyse des sciences sociales, ils acquièrent, en revanche, rapidement les principes techniques d'intégration paysagère des opérations sylvicoles. A la fin du cycle de formation, les agents sont donc en principe capables d'instruire des dossiers à caractère paysager, de résoudre des problèmes simples sur le plan technique et, dans des cas plus complexes, de préparer un cahier des charges à l'attention d'un bureau d'étude spécialisé.

\section{Les points à améliorer}

Un point important n'a jamais été clairement exposé par le commanditaire des stages qui est celui de la relation de la formation au temps tel que l'exprime Bel et coll. (1988, cité dans Dubar, 2000): La DERF attendait-elle du programme de sensibilisation une efficacité à court terme permettant de gérer les problèmes au quotidien, un accompagnement du changement à moyen terme initié par un choix stratégique ou une anticipation à long terme en vue de préparer des changements futurs et incertains ? De fait, vu sa structuration en trois sessions, le programme d'action vise ces trois logiques à la fois. La prise en compte du paysage dans la gestion forestière parait inéluctable à long terme au vu des attentes actuelles de la société ; c'est un point sur lequel la première session insiste particulièrement. Quant à la mise en œuvre concrète d'actions paysagères au quotidien, elle est dès aujourd'hui une réalité à laquelle les forestiers sont confrontés et à laquelle la troisième session apporte des éléments de réponses.

Cette ambiguïté sur la relation au temps s'est manifestée concrètement dès la fin des cycles de formation. La DERF a invité ses agents à se former dès 1996, l'ensemble du programme était achevé en 1999, mais elle a attendu deux ans avant de leur reconnaître, de manière officielle, une compétence spécifique. Celle-ci n'est intervenue qu'en mai 2001 à l'occasion de la parution du volet environnemental de la réforme des aides forestières de l'État. Dès lors, les agents formés ont été reconnus comme "des hommes de l'art pour ce qui concerne la rédaction d'une étude paysagère ou de son appréciation » (DERF, 2001). Mais l'impulsion qui aurait pu être donnée au réseau censé se constituer juste après les formations s'est trouvée amoindrie par ces deux années de délai. La DERF a, semble-t-il, attendu des réformes forestières importantes, notamment sur le plan environnemental, avant d'afficher les compétences nouvellement acquises par certains de ses agents. L'a-t-elle fait pour optimiser son investissement dans la formation, pour accompagner des réformes en cours, ou anticiper un changement à 
long terme ? Cette attente de la DERF peut aussi s'expliquer par la complexité de la notion de paysage qui, de par sa dimension sociale, reste un objet d'intervention publique plus difficile à expliciter dans une circulaire qu'une mesure technique d'ordre sylvicole et qui s'avère aussi plus difficile à mettre en œuvre sur le terrain.

L'autre point que la formation aborde sans apporter actuellement de véritable solution " clef en main » aux stagiaires, concerne la prise en compte des aspirations paysagères des populations locales. Certes, il n'a jamais été question que les agents traitent directement cette dimension des problèmes de paysage. Ils possèdent toutefois des éléments de réflexion et de langage leur permettant de dialoguer avec des professionnels compétents. Mais un problème demeure: les agents éprouvent de sérieuses difficultés à trouver des bureaux d'études capables d'aborder cet aspect social de la question. L'approche paysagère des bureaux d'études spécialisés est surtout d'ordre esthétique ou environnementaliste. Dès lors, ils intègrent peu ou pas, ou de façon incomplète, les données issues des approches sociologiques - quand bien même ces données sont disponibles. À l'inverse, seuls quelques bureaux d'étude de paysage ont une compétence dans le domaine de la sylviculture et de la gestion forestière. Là aussi, l'acquisition du langage technique particulier du forestier paraît un préalable nécessaire à tout paysagiste souhaitant travailler en forêt. De même que s'est posée la question: "Qu'est-ce que le paysage pour un forestier du Ministère de l'Agriculture ? ", peut aussi se poser : "Qu'est-ce qu'une forêt pour un paysagiste ou un agent du Ministère de l'Environnement?».

\section{Vrai partenariat ou partenariat mesuré ?}

La mise en place du programme d'action révèle des gradients de partenariat différents entre ministères et entre agents formés.

Au niveau des ministères, le partenariat est à la fois perçu comme un rapprochement stratégique inéluctable vu les proximités thématiques, mais aussi comme un frein à l'initiative de chacun, voire comme un empiètement de son pré-carré. Il ne s'agit pas, pour eux, de remettre en cause la sensibilisation conjointe de leurs agents à la forêt et au paysage. En revanche, l'affichage de nouvelles compétences dans un domaine réservé jusqu'à présent à l'un ou l'autre acteur, peut inciter à un partenariat mesuré. Cela explique peut-être le faible nombre d'agents formés venant des services déconcentrés du Ministère de l'Environnement d'une part et le délai que la DERF s'est donnée avant d'afficher les nouvelles missions de ses agents en matière de paysage.

$\mathrm{Au}$ niveau des stagiaires, la formation permet d'échanger les savoirs respectifs de chacun en matière de sylviculture et d'environnement et de mieux appréhender les pratiques professionnelles des uns et des autres. Les agents en formation élaborent des solutions communes et négociées lors des sites-ateliers, mais les situations d'adhésion voire de connivence entre agents du Ministère de l'Agriculture et ceux de l'Environnement sont plus rares. Les logiques institutionnelles ressurgissent inévitablement lors des discussions. Il convient alors de leur rappeler que la légitimité en matière de paysage n'appartient pas plus à l'un ou à l'autre groupe professionnel et que bien d'autres groupes sociaux peuvent se prononcer sur le sujet. 


\section{Conclusion}

31 Cette action de sensibilisation "Forêts-Paysages " souligne une inflexion majeure prise par la DERF dans la prise en compte d'une question sociale en émergence. Dans le domaine de l'appui technique, cette redéfinition des termes du débat a des répercussions sur le contenu des formations au paysage forestier. Tout d'abord, les actions de formations sont passées d'une à deux journées d'initiation en 1980 à près de douze jours de sensibilisation approfondie aujourd'hui ce qui montre l'investissement et l'intérêt croissant de la DERF pour cette problématique. Ensuite, ces formations ne sont plus réservées aux seuls forestiers, mais elles sont aussi ouvertes aux personnels du Ministère de l'Environnement. Enfin, les formations n'apportent plus seulement des " recettes techniques prêtes à l'emploi », mais aussi des outils et des méthodologies de travail permettant d'appréhender la question du paysage forestier par étape, dans sa dimension technique et sociale.

Néanmoins des difficultés persistent. En premier lieu, si les méthodes et les résultats de la recherche sur la perception de la forêt par le public peuvent être présentés à l'occasion des formations, ils ne sont pas directement transposables en actions concrètes par les agents. Ensuite, l'étape opérationnelle de la négociation et de la construction d'un projet en commun reste très longue. Et, c'est seulement après ces deux étapes que les réponses techniques vues lors de la troisième session peuvent être appliquées. Cette rupture est aggravée par la faible connexion entre la recherche d'une part et les paysagistes professionnels d'autre part. Le positionnement des Ministères et des centres de recherches sur ces questions devrait aider à combler ce fossé. Si les méthodologies sont affinées et leurs transferts en cours, il faudra encore que les professionnels de la forêt et du paysage se les approprient plus largement. La question du niveau d'anticipation de ces formations est donc centrale pour le commanditaire de l'action et le responsable pédagogique : il s'agit de trouver un équilibre entre, d'une part, une formation trop anticipatrice et prospective, éloignée des problèmes concrets $\mathrm{du}$ stagiaire et pour laquelle les savoirs sont loin d'être établis et, d'autre part, une formation trop opérationnelle, au contenu peu novateur et qui n'infléchirait pas assez les pratiques professionnelles des agents eu égard aux enjeux fixés par le commanditaire.

\section{BIBLIOGRAPHIE}

Barthod, C. (1995). Le débat international sur la gestion des forêts. Aménagement et Nature, $\mathrm{n}^{\circ} 115$, 65-80.

Berque, A. (1995). Les raisons du paysage. De la Chine antique aux environnements de synthèse. Paris : Éditions Hazan.

Breman, P. (1997). Cahier de recommandations à l'usage des sylviculteurs pour une approche paysagère de la production en forêt morvandelle. Dijon : Préfecture de la Région de Bourgogne. 
Breman, P. (1993). Approche paysagère des actions forestières, guide à l'attention des personnels techniques de l'Office National des Forêts. Fontainebleau : Cemagref Nogent/V et ONF-DTC.

Coll. (1995). Paysage au pluriel. Pour une approche ethnologique des paysages. Paris : Éditions de la Maison des sciences de l'homme, Collection Ethnologie de la France, Cahier $n^{\circ} 9$.

Coll. (1991). De l'agricole au paysage. Études rurales, $\mathrm{n}^{\circ}$ 121-124.

DERF. (2001). Conditions de financement, par le budget général de l'État, des projets d'investissements forestiers ou d'actions forestières à caractère protecteur, environnemental et social. Paris : Ministère de l'Agriculture, Sous-Direction de la Forêt, circulaire DERF/SDF/C2001-3010.

Deuffic, P. (1996). Perception des impacts paysagers des actions forestières. Ingéniéries EAT, $\mathrm{n}^{\circ} 7$, 31-39.

Dubar, C. (2000). La formation professionnelle continue. Quatrième édition. Paris : Éd. La Découverte, coll. Repères.

ENPDD. (1995). Le diagnostic du territoire. Document de travail. Rambouillet : Centre National de Ressources Agriculture-Environnement.

Freytet, F. et Rumelhart, M. (2001). Les forestiers, fabricants de paysage. Forêt Entreprise, $\mathrm{n}^{\circ} 140$, 28-33.

Luginbühl, Y. (1993). Bilan et prospective de la politique paysagère de la DERF. Paris : Ministère de l'Agriculture, École Nationale Supérieure du Paysage de Versailles.

Luginbühl, Y. (1991). Le paysage rural. La couleur de l'agricole, la saveur de l'agricole, mais que reste-t-il de l'agricole ? Études rurales, $n^{\circ}$ 121-124, 27-44.

Micoud, A. (1991). Des Hauts-Lieux. La construction sociale de l'exemplarité. Paris : Édition du CNRS.

Ragouet, P. (2000). Les approches du paysage en sciences sociales. Différenciations conceptuelles et disciplines. Rapport post-doctoral, Cemagref, Bordeaux.

SOFRES. (2001). Les Français, la forêt et le bois. Paris : SOFRES. [En ligne 09-10-01]. Accès : http// www.sofres.com/etudes/pol/291100-bois-n.htm

\section{NOTES}

1. Communication orale de C. Barthod, Sous-directeur des Forêts au Ministère de l'Agriculture.

2. L'appellation générique de paysagiste recouvre deux principaux diplômes de nature différente, dont celles de :1) paysagiste diplômé par le Gouvernement (paysagiste DPLG). Ce diplôme est délivré par des écoles nationales supérieures sous tutelle du Ministère de l'Agriculture, du Ministère de la Culture ou du ministère de l'Éducation nationale ;2) ingénieur de l'Horticulture et du Paysage, diplôme délivré par l'Institut National d'Horticulture sous tutelle du Ministère de l'Agriculture. 


\section{AUTEUR}

\section{PHILIPPE DEUFFIC}

Philippe Deuffic est ingénieur d'étude au Cemagref (Institut public de recherche pour l'agriculture et l'environnement), l'auteur travaille sur les représentations sociales de l'espace rural et les attentes de la société en matière de paysage. Dans le cadre du transfert des résultats de la recherche, ces travaux alimentent depuis 1996 un programme de formation continue sur le thème de « la prise en compte du paysage dans la gestion forestière » mené à l'attention des agents du Ministère de l'Agriculture. 